\title{
Biochemical changes in burned patients
}

\author{
Pamela Hinton \\ M.B., Ch.B., D.R.C.O.G., M.R.C.P. \\ Renal Unit, Royal Postgraduate Medical School, London, W.12
}

\section{Summary}

\section{Some of the biochemical changes that may occur after} severe burns are described.

Not all of them require active therapy, and attempts to correct them may be more harmful than beneficial to the patient.

OF the problems associated with anaesthesia and surgical treatment in burned patients, some are the result of secondary biochemical changes or indiscriminate attempts to reverse such changes (Hutchins, McLaughlin \& Hayes, 1961; Stoner, 1969, 1970).

For example, one of the most constant, if least striking, early features of the metabolic response to injury-including burn injury and surgical traumais a temporary fall in plasma potassium levels. This change may cause cardiac problems for the very elderly, those with pre-existing cardiac disease, and those who are total body potassium-depleted. It is certainly very dangerous for any patient who has been treated with digoxin because it potentiates the actions of this drug, and may, therefore, promote severe arrhythmias such as complete heart block. Trouble can te avoided by very cautious use of digoxin and the addition of some potassium to any fluids given during and immediately after operation(Fig. 1).

Hypoglycaemia is also one of the biochemical changes which can cause problems more frequently than is generally realized, in severely burned patients, and those with pre-existing liver function disturbances (Fig. 2). Severely burned patients are likely to be glycogen-depleted within about $8 \mathrm{hr}$ after injury, their energy requirements are extremely high, they rapidly develop severe calorie-protein malnutrition, and frequently they develop liver function abnormalities. Such patients are poorly equipped to compensate for even a short period of fasting, and may become severely hypoglycaemic during or after operations. Prophylactic infusions of carbohydrate can prevent this complication, and the fact that such patients are pseudo-diabetic is not a contra-indication to such treatment, it is an indication for insulin therapy if hyperglycaemia demonstrates that they are not utilizing the carbohydrate (Fig. 3a and b).

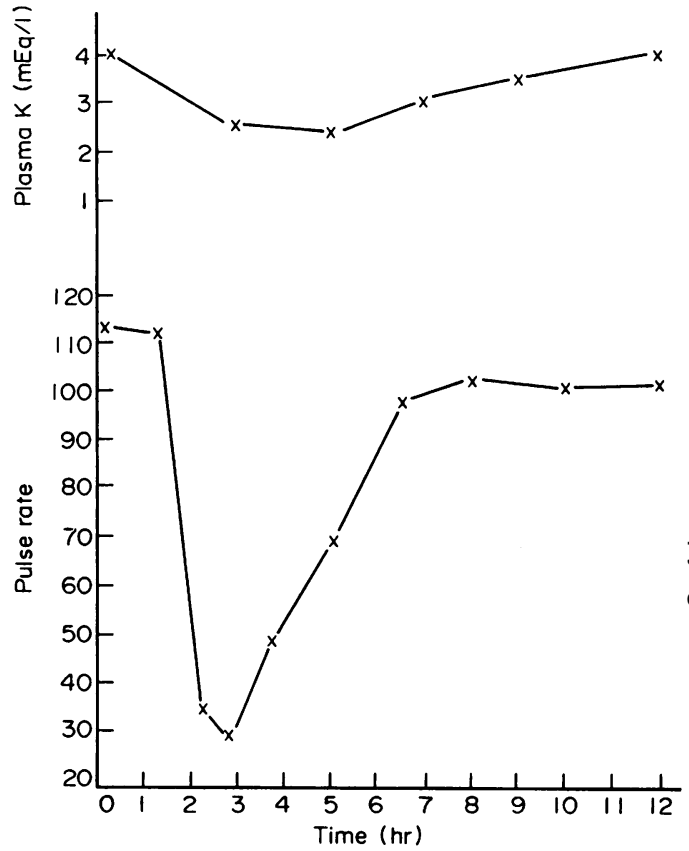

Fig. 1. One of the least striking effects of the nonspecific stress reaction, a fall in plasma $\mathrm{K}^{+}$concentration, can have very serious consequences for patients being treated with digoxin. This patient, who was admitted with $20 \%$ burn injuries developed complete heart block which was rapidly corrected by infusions of potassium.

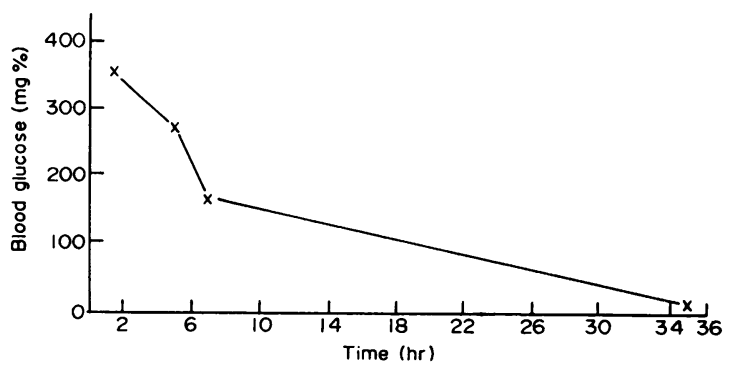

FIo. 2. Hypoglycaemia after stress. J.D., 22 years, RTA. 


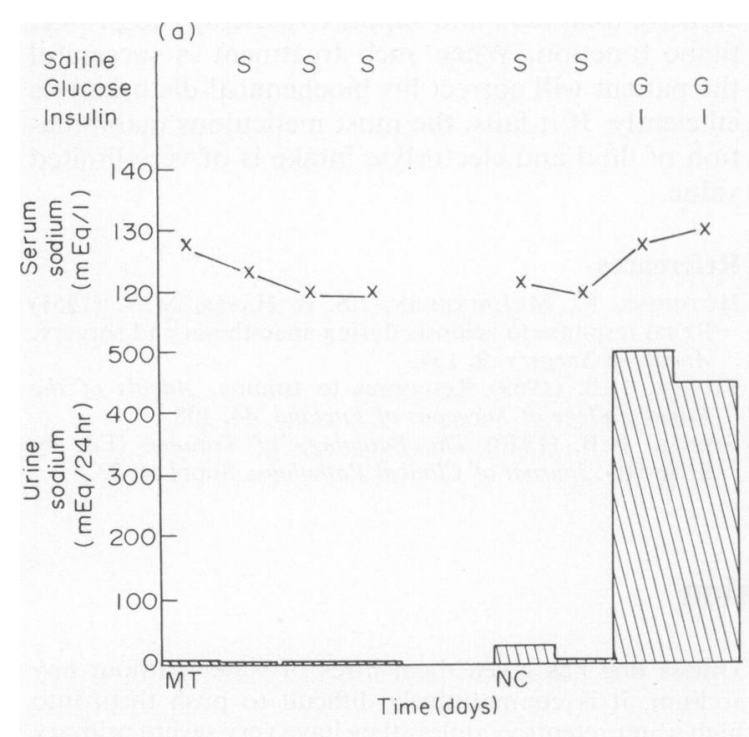

(b)

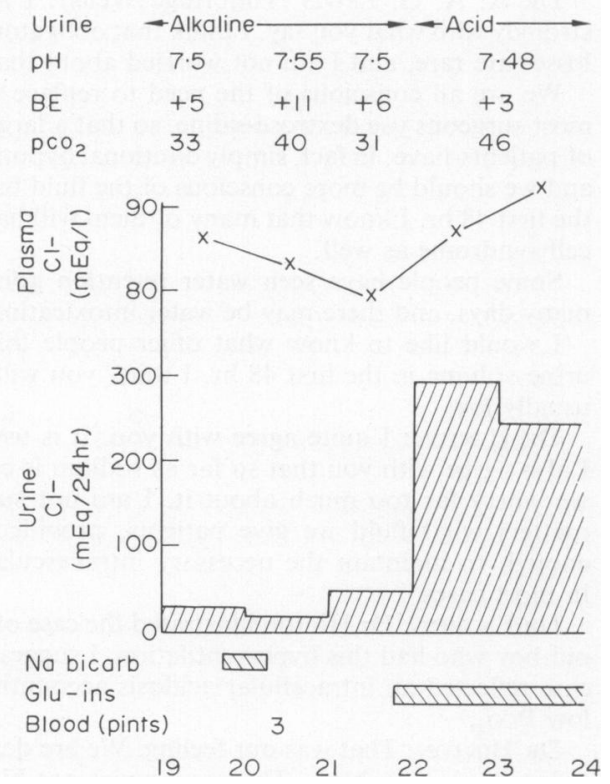

Fig. 3 Changes in two young patients both of whom had $60 \%$ burns. Both became severely hyponatremic and exhibited marked renal sodium retention, possibly as a result of the development of the 'sick-cell syndrome'. (a) When this is the case, treatment with ever increasing amounts of sodium may be of no benefit. Attempts to promote a redistribution of fluid and electrolytes by using glucose and insulin may be of greater benefit, clinically and biochemically. (b) Effects of a glucose-insulin infusion. KH., 9 years, $65 \%$ burns.
Hyponatremia, metabolic acidaemia and hyperventilation with a fall in $\mathrm{PaCO}_{2}$ levels are examples of changes which may cause problems per se, but may also cause problems if indiscriminate attempts are made to correct them.

Hyponatremia is a common finding in burned patients, but it is not always the cause of associated complications such as convulsions. Severely burned patients may exhibit polyuria and secondary aldosteronism for weeks, and there is good reason to suppose that they become potassium-depleted and magnesium-depleted. Treatment with sodium supplements will be of little benefit when cerebral complications are the result of magnesiumdepletion or hypoglycaemia.

A low plasma sodium level is not invariably the result of inadequate replacement of sodium losses or a disproportionate increase in the extracellular water content. It may also be a reflection of maldistribution of electrolytes and water if the patient has developed a 'sick-cell' syndrome. When this has occurred treatment with sodium supplements, drastic restriction of the water intake or treatment with diuretic drugs can do more harm than good even if it does result in a rise of the plasma sodium level. The correct treatment in such cases is rapid identification and correction of the factors which have caused cell membrane or cell energy production disturbances, such as hypoxia, underperfusion, or severe malnutrition.

Profound disturbances in hydrogen ion production and excretion occur in stress. A metabolic acidaemia always causes concern, but before attempts are made to correct it, attempts should be made to determine whether it is a reflection of pre-existing renal insufficiency, a common finding in the very elderly, and not a rare finding in the general population. Patients with chronic renal failure are well adapted to living with a metabolic acidaemia, and often are hypocalcaemic. Rapid infusions of sodium bicarbonate may correct their acidaemia, but also promote severe hypertension and marked tetany.

Hyperventilation and a reduction in $\mathrm{PaCO}_{2}$ levels may reflect increased hydrogen ion production as a result of hypercatabolism in association with abnormalities in buffering ability and renal tubular abnormalities, such that urine hydrogen ion excretion is reduced. It may be a vital compensatory response to retention of hydrogen ion at an intracellular level, and not only at an extracellular level if the patient is also potassium depleted or has developed a 'sick-cell' syndrome. Despite the fact that hyperventilation results in increased water loss and a rise in energy requirements, it may not be advantageous to promote respiratory centre depression, and one of the reasons why burned patients sometimes die suddenly if given relatively small doses of 
narcotic drugs may be that such drugs prevent them from compensating in the only way they can for acidosis.

These examples illustrate the point that there is good reason to employ the golden rules governing the management of secondary biochemical changes when treating burned patients. These are that the significance of biochemical changes should be assessed in the light of all available information, including knowledge of all drugs which have recently been given. Secondary biochemical changes should be deliberately corrected only if there is good reason to suppose that they are dangerous per se, and close observations should be made to detect any unexpected consequences of biochemical meddling.
Preferably, attention should be concentrated upon curative treatment and supportive therapy to protect tissue function. When such treatment is successful the patient will correct his biochemical disturbances efficiently. If it fails, the most meticulous manipulation of fluid and electrolyte intake is of very limited value.

\section{References}

Hutchins, P., Mclaughlin, J.S. \& Hayes, M.A. (1961) Renal response to acidosis during anaesthesia and surgery. Annals of Surgery, 9, 154.

StONER, H.B. (1969) Responses to trauma. Annals of the Royal College of Surgeons of England, 44, 308.

Stoner, H.B. (1970) The Pathology of Trauma. (Ed. by S. Sevitt). Journal of Clinical Pathology. Suppl. 4, 23.

\section{Discussion}

DR LAIRD: We had a patient at Chepstow about a year ago who puzzled us greatly because every time she was anaesthetized she collapsed and came nigh unto death, and recovered when given $100 \mathrm{mg}$ of hydrocortisone sodium succinate in spite of having plasma cortisol of the order of $20 \mu \mathrm{g} \%$. I wonder whether she was in fact a hypoglycaemia and we corrected her hypoglycaemia by giving her cortisone.

DR HINTON: I think it is certainly possible. On the other hand, there is considerable variation in the patients' abilities to produce the optimum cortisone levels which they need.

DR P. BASKeTt (Bristol): I wonder whether Dr Hinton could give us some clinical guidance on how to diagnose whether a low serum sodium is due to loss of sodium or whether it is all disguised as a high serum potassium, and how we are to treat this?

DR HINTON: I do not know of any one sure method, but I think there are two or three clinical observations and biochemical measurements which one has to consider together. The first is that the truly depleted patient, unless he has severe renal failure and cannot excrete fluid at all, usually looks clinically dehydrated and the 'sick-cell' patient usually looks oedematous.

The second is that as far as I am concerned, unless the patient has very severe renal failure and is an obligatory salt-loser, the sodium-depleted patient does not often have a low sodium level. They may drop to something like $135 \mathrm{mEq} / \mathrm{l}$ but in fact much more commonly they have a relatively high sodium level. We see this particularly in our patients with chronic renal failure. Obligatory salt-losers come in desperately salt-depleted, and almost invariably they have increased sodium levels-not decreased levels. Very severe hyponatremia one sees only in patients who have become severely salt-depleted, and then have been loaded with fluid without salt.

With respect to burned patients, it is very difficult to measure exactly what fluid a patient has had and exactly what he has lost. However, the maximum sweat sodium one sees in patients is less than $60 \mathrm{mEq} / \mathrm{l}$. When they are evaporating fluid they are losing water and not salt.
Unless one has given them litres of water without any sodium, it is comparatively difficult to push them into high water retention, unless they have very severe primary renal disease.

DR A. A. G. Lewis (Tunbridge Wells): I agree very strongly with what you say. I think that obligatory sodium losses are rare, and I am not worried about that.

We are all conscious of the need to replace fluid, ando most surgeons use dextrose-saline, so that a large number of patients have, in fact, simply dilutional hyponatraemia and we should be more conscious of the fluid balance for the first $\mathbf{4 8} \mathrm{hr}$. I know that many of them will have a sickcell syndrome as well.

Some people have seen water retention going on for many days, and there may be water intoxication.

I would like to know what other people think about urine volume in the first $48 \mathrm{hr}$. I think you will find it is usually low.

DR Hinton: I quite agree with you. It is usually low. I also agree with you that so far as sodium is concerned, we worry far too much about it. I am not sure that it matters what fluid we give patients, provided we give enough to maintain the necessary intravascular volume in most cases.

DR ENTRESS: Dr Hinton illustrated the case of a 9-yearold-boy who had this hyperventilation. I suggest that this case reflected an intracellular acidosis accounting for his low $\mathrm{PCO}_{2}$.

DR HINTON: That was our feeling. We are dealing with a distribution problem. The answer was not bicarbonate but trying to get at the cause of the distribution problem. We see it in a great many of our patients.

DR Rowell: Could I refer to the anaesthetized patient we saw on the first slide. The pulse dropped to 30. Did she recover of her own accord or was she treated?

DR HINTON: We gave her potassium. In fact, we were very worried and we were making arrangements for intravenous pacing, but as soon as we gave her potassium she reverted to her usual rhythm.

Dr J. Murray (East Grinstead): I would like to ask, with reference to the 9-year-old boy of whom we have 
just been speaking whether cardiac output determinations have been done? With his low $\mathrm{PCO}_{2}$, the cardiac output would fall by half. This would further increase the metabolic acidosis and it could in fact cause the metabolic acidosis.

DR HINTON: In that particular case we could not do the cardiac output. We were doing some blood volume studies on these patients and we found that they were very much lower than we had suspected. You will find in the oedematous patient incredibly low total blood volumes and red cell volumes. From some animal work I have done I suspect that this is a significant factor and one that we do not take into account at times.

DR Thomas: I understood Dr Lewis to say that one should not be worried about a low urinary flow in the first $48 \mathrm{hr}$. The urinary flow is one of the most valuable measures that we have as to how a patient is progressing, as regards his hydration. Could Dr Hinton give a rough idea as to what is a low exceptional figure for urinary output?

DR Hinton: The urine volume is important, not because it may or may not indicate acute renal failure, but as a very useful monitoring tool.

What I would be interested in is not simply the actual urine volume but whether it reflects simply a physiological response to inadequate fluid with well maintained renal function, in which case one can afford to delay fluid replacement if one is worried about cardiac function and one does not want to give fluids fast.

If, on the other hand, it is a low urine volume-and I would call anything much less than $600 \mathrm{ml}$ in $24 \mathrm{hr}$ as something to worry about anyway-I would be very worried if the patient is showing a reduction in renal concentrating ability; the patient I would worry about is the one going on to acute renal failure and who I would take risks with. I would be happiest if I saw a patient excreting about $1500 \mathrm{ml}$ of urine in the first $24 \mathrm{hr}$.

DR LewIS: I do not think I have ever heard this question better answered!

QUESTION: I have one minor point: $600 \mathrm{ml}$ in $24 \mathrm{hr}$ or 1500 in $24 \mathrm{hr}$. Would you wait $24 \mathrm{hr}$ ?

DR Hinton: I would think that if it was a minor thing, if the patient had come in simply for a hernia operation, or something small, I would not like to justify putting a catheter in. If there is severe trauma I think the risks of inserting a catheter are well worth it to measure urine volume. I would much prefer to measure it hour by hour. If I see less than $20 \mathrm{ml} / \mathrm{hr}$, I think that is the time to interfere.

DR LEwIS: It is what is in it that matters. The specific gravity will give you some indication of good renal function. If it is highly concentrated, or if the urea is very high, that is an indication; but urine sodium does not help you, of course, because of the sodium retention at the same time. 